\title{
A systematic study of young multipolar planetary nebulae
}

\author{
Chih-Hao Hsia and Sun Kwok \\ Department of Physics, The University of Hong Kong, Pokfulam Road, Hong Kong, China \\ email: xiazh@hku.hk; sunkwok@hku.hk
}

\begin{abstract}
We have analyzed the HST images of ten young planetary nebulae (PNe) and found them to possess multipolar structures. Concentric arcs and 2-D rings are also found in some objects.
\end{abstract}

Keywords. planetary nebulae, general, stars: AGB and post-AGB

\section{Introduction}

The morphological transformation from spherical geometry to bipolar and multipolar structures of PNs is believed to occur shortly after the asymptotic giant branch (AGB). Recent high-resolution narrow-band observations of young PNs have revealed complex, multipolar structures in objects such as He 2-47, M 1-37 (Sahai 2000) and NGC 6644 (Hsia et al. 2010). In this paper, we study ten young PNs (H 1-54, He 2-86, He 2-320, He 2-447, IC 5117, M 1-30, M 1-59, M 1-61, M 3-35, and NGC 6790) with the goal of determining their nebular structures.

\section{Observations and results}

The observations are based on two young PN surveys (GO 8307, PI: S. Kwok and GO 8345, PI: R. Sahai) using the HST Wide Field Planetary Camera 2 (WFPC 2) with three narrow-band filters (F502N ([O III $]$ ), F656N (H $\alpha$ ), and F658N ([N II $]))$. The actual observations were made with different exposures to allow for the imaging of both bright central region and faint outer parts. The composite-color HST images of four of our sample objects (He 2-320, He 2-447, M 1-30, and M 1-61) are shown in Fig. 1. Multiple lobes are commonly found in the objects. The multiple structures of PNs are usually interpreted as the result of simultaneous collimated outflows in different directions, or the outflow direction has changed with time. An alternative explanation is that the multiple lobes are the results of illumination and holes in the equatorial torus (Kwok 2010). In addition, a concentric arc and several 2-D rings are found in He 2-320 and M 1-30 (Figs. 1a and 2), respectively. These structures are likely to be the result of interactions between a time-variable fast wind with the remnant of the AGB circumstellar envelope .

\section{Acknowledgements}

This work was supported from the grant of Research Grants Council of the Hong Kong Special Administrative Region, China (Project No. HKU 7031/10P).

\section{References}

Hsia, C.-H., Kwok, S., Zhang, Y., Koning, N., \& Volk, K. 2010, ApJ, 725, 173

Kwok, S. 2010, PASA, 27, 174

Sahai, R. 2000, ApJ, 537, L43 

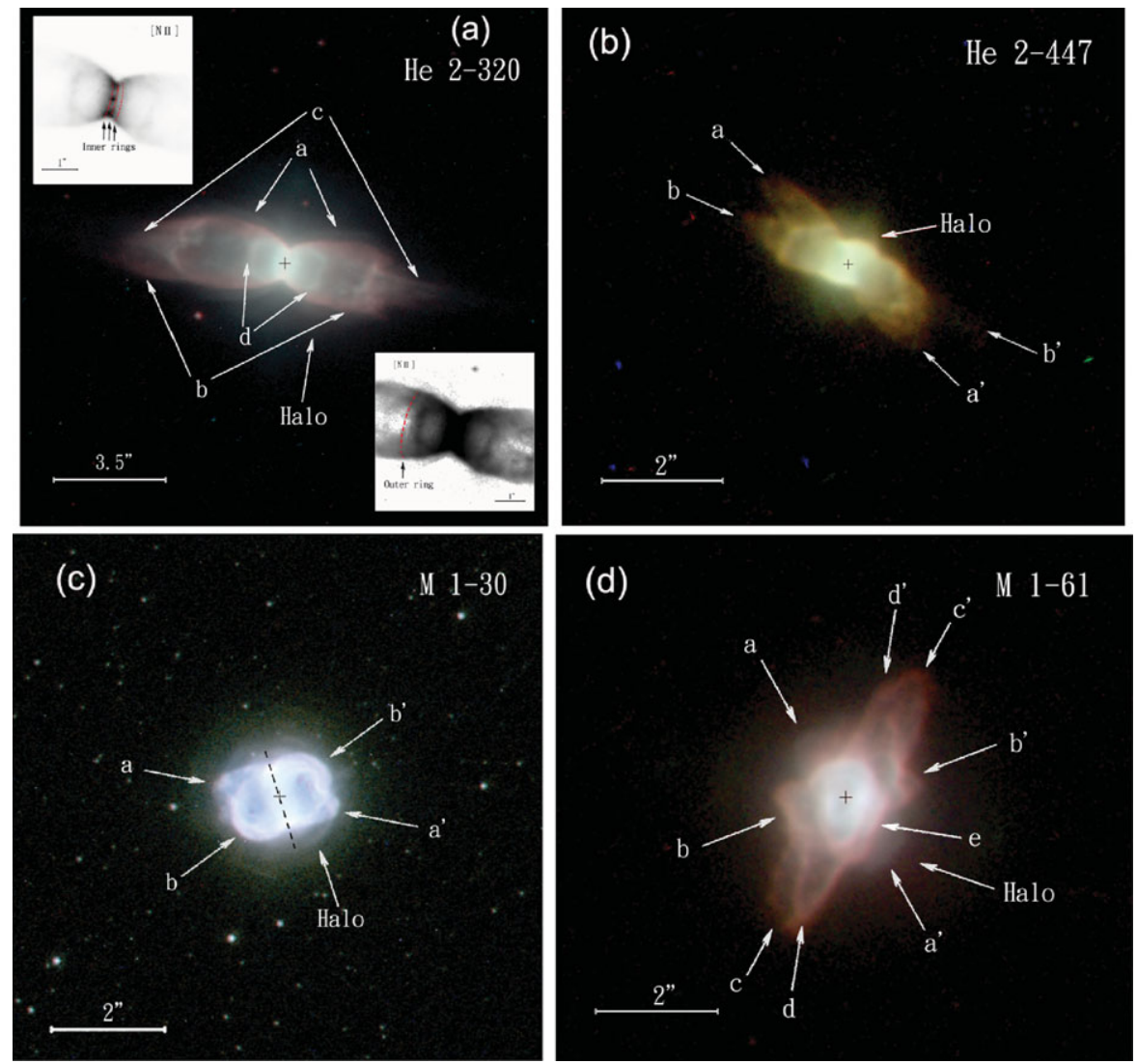

Figure 1. Composite-color images of four compact PNe. The [O III] images are shown as blue, the $\mathrm{H} \alpha$ as green, and the [N II] as red. The central star positions are marked as crosses and their various lobes are labeled by letters $\left(a, a^{\prime}\right.$, etc.). The black dotted line shows the minor axis direction in the image of M 1-30. Four ring structures (red dotted lines) can be seen in enlarged $[\mathrm{N}$ II] images of He 2-320. The central nebulosity in the image of M 1-61 (marked as $e$ ) could be the projection of another pair of lobes aligned almost along the line of sight.

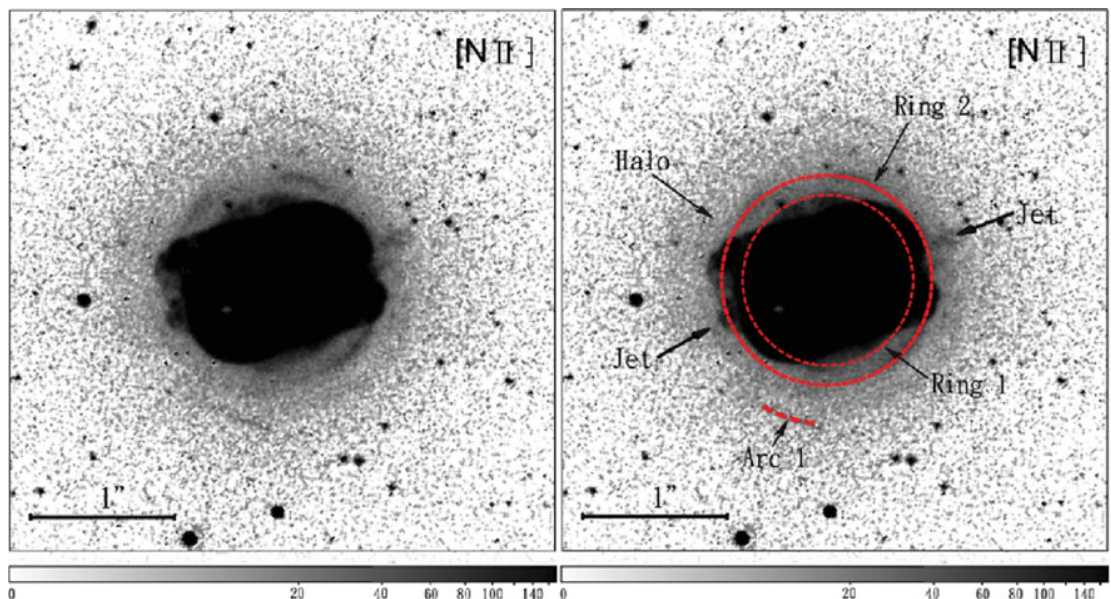

Figure 2. HST WFPC 2 [N II] images of M 1-30 after the application of an unsharp mask. Two rings and a arc structure are marked as red dotted lines. A pair of jets can also be seen. 